FengShui and Library Building : An introductory approach

\author{
--I.P. Adhikari, \\ iadhikari@hotmail.com
}

\section{Origin of FengShui:}

Similarly, five thousand years ago the Chinese wisemen propounded a philosophy and practice that is now known to the world as FengShui. FengShui believers opine that Qi (pronounced chee) energy is the animation life-force that exists everywhere. They say Chee (or Ch'i) energy is positive when allowed to flow freely, negative when left to stagnate in nooks and crannies of our homes and office rooms. FengShui is used to decide location, construction and other architectural features of buildings, the placement and style of furniture, interior/exterior decoration and even the location of plantings so that Chee energy has free movement in and around the building. By doing so it is believed that the positive elements that protect us are accentuated and those that are harmful or negative are minimized. As a result, the followers may lead a life of contentment, happiness and abundance.

The word FengShui itself is a Chinese word and literally means wind and water. Air and water are the two prime matters for our very survival. FengShui is based on Taoist philosophy of Yin and Yang. The ancient Taoists used the symbols of 'Yin ' and 'Yang', i.e. the two opposites in the universe, viz. Female and Male, Negative and positive, Night and day, etc. These two opposites are said to be complement to each other and none of these can exist without the other. The followers opine that we need a perfect balance of Yin and Yang in our life too. Yin and Yang are represented by a circle, a symbol of the universe in which two tadpoles, one black and the other white, are seen living together in perfect harmony. (We can see the symbols of Yin and Yang in the national flag of South Korea.)

\section{VastuShastra vs. FengShui:}

VastuShastra is often termed as the elder sister of FengShui. The word 'VastuShastra' is a combination of two Sanskrit words viz. Vastu and Shastra. Vastu means a house/building and Shastra means scripture/science. In a modern sense it's called Edifice science. As the name VastuShastra is a Sanskrit word so we can infer that the culture of VastuShastra has its roots in Hindu religion and culture. VastuShastra is said to have originated long ago in the Vedic era some 7,000 to 15,000 years ago. It is a Hindu system of philosophy based on the belief that we can improve our lives by rearranging the spaces or things where we live and work.

Long long ago, during the Mahabharata period, as some Hindu scriptures says, the city Indraprastha was built as per the rules of VastuShastra. Similarly the city of Dwarka was planned and designed by Bishwokarma, the wellknown architect of that time. He built the city following the principles of VastuShastra. So, the tradition of VastuShastra has long roots and is still being widely practiced in India.

Both FengShui and VastuShastra practices are based on a set of principles. Vastu philosophy has used the five basic elements viz. space, air, fire, water and earth. FengShui has also used five basic elements replacing the VastuShastra's air and space with metal and wood. They both aim at removing obstructions so that energy can flow freely thereby creating environmental harmony. Both practices have been gaining worldwide popularity even in this age of science and technology. The two approaches have similar goals. Both of them teach us to live in harmony with the nature.

\section{Popularity and Ubiquity of FengShui:}

Even though FengShui appeared thousands of years after VastuShastra, the popularity of FengShui seems to have spread many folds than 
that of VastuShastra. Nowadays the presence of FengShui practice can be felt in almost all over the developed countries in Europe, US, Australia etc. FengShui practice has even invaded the place of origin of VastuShastra i.e. India. The constructors of residential or business office buildings or even the general folks of urban India have started applying FengShui techniques \& remedies in addition to VastuShastra

Hong Kong is very famous in FengShui culture. FengShui experts say that Hong Kong itself is situated in a naturally good FengShui position, that's why she has made astounding progress in business and commerce and has become a business hub in Asia. Secondly its people apply FengShui very much during or before the making of their new homes and business/office complexes. Even if their homes are not in accordance with FengShui principles then they resort to FengShui remedies to correct the mistakes. Jokingly one author has mentioned the scenes of many places where mirrors could be seen placed on the outside walls of the homes of Hong Kong citizens. On seeing this, the author felt as if there was a mirror war in the neighborhood. Later, he found out this practice of mirror hanging on outside walls was done simply to ward off the evil eyes (often called the poison arrows) symbolically, according to FengShui culture.

The HSBC (Hong Kong Shanghai Banking Corporation) building in Hong Kong is one of the many buildings that is said to have followed FengShui principles. Even the elevators ${ }^{1}$ of the HSBC building are said have been constructed in accordance with the FengShui principles. Outside Hong Kong, the presence of FengShui can be seen vividly in the United States. In the US alone, dozens of books have been written on FengShui. Many consultants have succeeded in selling their FengShui ideas to the very modern people. The FengShui consultants have even found the opportunity to air their FengShui related ideas through T.V shows and many websites can be found exclusively devoted to FengShui.

Actually the popularity and ubiquity of FengShui is attributed to its simplicity, because its principles are comparatively very simple than that of VastuShastra. In addition, FengShui gives

\footnotetext{
${ }^{1}$ National Geographic TV channel, " The Megacities,
} ...HongKong." emphasis on beauty, comfort and functionality. That's the main reason behind its popularity.

\section{Library's success and FengShui:}

A library is established with the objective of serving the people. Its success is measured by its popularity, the frequency of library users' visits per year and by means of making the most of its holdings. Collecting volumes of books, rendering variety of services and employing trained librarians are not enough. EVEN A WELL STOCKED LIBRARY WHICH RENDERS A VARIETY OF SERVICES WITH WELL TRAINED LIBRARIANS is bound to succeed or fail on the following grounds.

\section{Layer I: Approachability:}

Libraries are intensively used. And the more it is visited and used the more it is termed successful. Therefore, it is better that a library building must be constructed or housed at a centrally located place so that maximum number of persons could approach the library with ease. But it doesn't mean that it should be located near to a potentially heavily crowded place. A place where there is always commotion is against the sentiment of FengShui. On the other hand if a particular library is located very far away or not at an easily approachable place, even if it has very good stock of books with best services the library cannot draw maximum number of people as expected. In this case, most of the library holdings remain unused. And such a situation cannot be termed as success of the library.

For example, our Nepal National Library's location is considered in a remote area i.e. too far away from the city center. So, it is difficult to approach the library too often even in need. Secondly it has not its own building. It has occupied a few rooms of the ministry of general administration at Pulchowk. Thus, it tends to be totally dominated by the giant size of the ministry. Had the NNL been housed somewhere near the city centre, the number of visitors or users would have increased in many folds. Both VastuShastra and FengShui give importance on the right location of buildings whether it is a home or a business complex or an organization.

\section{Layer II: Beauty or aesthetic quality:}


From the time immemorial beauty has always been the enchanting force to human beings. Flowers, birds, greeneries, water ponds etc have always fascinated and soothed the human minds and senses. Many wars have been fought for the possession of beautiful things. It is a human desire to use or keep or watch beautiful things. Naturally, where there is beauty, we are attracted to it and tend to stay there longer. FengShui has always given emphasis on beautiful surroundings. We the librarians or the concerned persons too have to have a sense of aesthetic appreciation. FengShui emphasizes on creating pleasing atmosphere. People visit the library as naturally as they go into their own living rooms. So unless the environment is pleasing people do not generally go there or stay there for longer periods.

Symmetry is the prime factor in beauty. This world is amazingly symmetrical. Asymmetrical things look ugly and clumsy, be it an object or a person or building. If each of the two equal halves of anything looks like a mirror image of the other half then it is perfectly symmetrical. For example our two eyes or ears or hands etc are exactly symmetrical. Even if a single object, for example, our nose or any one of body parts (or any natural object) is imaginarily cut into two halves, we see that each of the two halves exactly resembles to the other, i.e. they look exactly symmetrical to each other. This same principle is applied to a building.

Secondly, FengShui advises to unclutter the room's space. Discard unnecessary items that have occupied the space causing hindrance or distraction. Library's space should be kept uncluttered by discarding unused and outdated books \& journals. Similarly, the furniture or equipment that is no longer in use must be removed from the spaces that could be better utilized in other ways. Thirdly, FengShui strongly advises to keep the rooms or other visible things and spaces neat, clean and tidy. All these factors enhance the beauty of a building and rooms.

\section{Layer III: Comfortability:}

Once the people find it easy to approach a library and get fascinated by its beauty, then they seek comfortable environment to remain there till they finish their objective of visiting that particular library. FengShui emphasizes on comfortability as much as the other previous two qualities. Well ventilated room(s) with enough light makes the readers feel comfortable upon entering the library and only then, they begin to think of finding a comfortable place to read books, journals etc. Therefore, the chairs and tables should also be comfortable and the combination of the sitting position on the chair and writing on the table should not make him/her feel uncomfortable.

Keeping this in mind and realizing the fact that beautiful things are not complete in themselves, many furniture manufacturers in developed countries have begun to produce furniture in accordance with ergonomic principles. Ergonomics is a study of designing of equipment and furniture in order to help people work more efficiently for longer periods. Ergonomically designed furniture or equipment greatly minimizes the risk of eye strain, backbone ache, legs' tiredness etc. But in developing countries, this principle does not seem to have been practiced or even known by the furniture manufacturers. Besides this, it is often found to our dismay or even to a ridiculous extent to see a chair's legs got broken as soon as a person sits on it or even gets his/her trouser torn by protruding nails from the old chairs. FengShui does not even allow us to use the tables or other furniture that have pointed corners, and advises us to use round tables and other round-cornered furniture instead. Not only chairs or tables but other equipments also need to be made comfortable.

\section{Layer IV: Functional quality:}

Next comes functional quality. It completes or fulfils the beauty and comfortability of anything. FengShui greatly emphasizes on functionality of a building or equipment inside a building. Non-functional equipments are in themselves symbols of bad FengShui. First of all library building architecture should be relevant to the user's requirements, relevant to the library's objective of holding the stock. Here relevant means functional. A beautiful library with comfortable furniture environment cannot serve the clientele well if the equipments in the library kept for use are not functional i.e. do not work well rather such a situation greatly irritates the users. They feel that using such non-functional equipment or materials is a mere wastage of their valuable time and energy. As a consequence they stop using them 
and leave the library without fulfilling their objective of visiting the library.

As said earlier such things are to be discarded or repaired as soon as possible so that these incomplete items would not distract or cause hindrance to the users. A computer or its program that does not operate well or a beautiful book that has missing pages or has out of date contents or a costly photocopy machine that does not work well etc. are some examples of nonfunctional items. So, functionality of library equipment is as important as a beautifully designed building with cozy interior environment.

\section{Layer V: Service attitude:}

Actually it has nothing to do with library building or equipment. However, it plays a vital role in attracting library users in making a library succeed in its endeavors. It is entirely an internalbeauty concept. As the clean tidy and beautiful environment is important so is a library staff's welcoming and helpful attitude. FengShui gives emphasis on a person's ability to be friendly to create congenial environment in the workplace. An employee who works in a grudging mood generates bad Chee and eventually dispels the potential users as well as the permanent ones. So, service attitude of the library staff is considered supreme.

\section{FengShui in Nepal and Libraries:}

Concerning Nepal, until less than a decade ago, FengShui was not known even to the urban educated people of Kathmandu. Nowadays the teenage youths in Kathmandu can be seen buying the FengShui items such as the idols of laughing Buddha, wind chimes, crystal balls etc in the local Tibetan shops. Some construction companies of civil homes in Kathmandu claim to have followed and applied the principles of both the FengShui and VastuShastra in their new buildings. And the educated people seem to have started taking interest in FengShui before they build their home.

In Nepal, there is no standard for a new library construction as yet. In the Govt. and University colleges, due to many reasons, (mainly economic of course) the libraries do not have their own building to house the books. The libraries occupy only a small room(s) of their parent body or housed in buildings primarily constructed for other purposes. People do not know that there is a library in the vicinity. Some libraries are set up near to a potentially very crowded place thus making prone to the disturbances from activities outside while some other libraries are set up at a far away inconspicuous place simply because the space or room was made available free of cost. In addition, the library rooms or shelves look cluttered, dirty and dusty. The spaces or equipment are not comfortable. Furniture and equipment are normally found non-functional. And obviously, all these things are against the sentiment of FengShui. These could be some of the reasons why such libraries are not frequented by most users.

\section{The Concept of Remedies:}

The FengShui experts opine that the bad effects of wrongly constructed buildings can be retarded by means of applying remedies. People buy these remedial ideas too but many people do not think that these remedies really work.

Hanging wind chimes on the ceilings, placing mirrors on the outside walls of the home (to ward off the poisonous arrows of the evil eyes), keeping miniature objects such as the image of laughing Buddha, tortoise, frogs, old coins, putting flower vases, keeping aquariums of gold fishes etc are some of the easy remedies that are prescribed by the FengShui experts to retard the evil effects of Bad FengShui construction.

But in reality, it is certain that the remedies do two good things. Firstly, the remedial items surely enhance the beauty of the surroundings and give us pleasant feelings. Secondly, they provide remedies at least symbolically, so that we could solace ourselves saying that remedies have been placed therefore no potential harm will come to trouble us. But actually it is not wise to construct a structure and later resort to the remedies. One writer says - "FengShui can not remedy a person or thing it can only bring the best out of what is already there".

\section{Conclusion:}

Even though being an ancient Chinese tradition, FengShui culture bears many useful and scientific reasons to be incorporated into our homebuilding practice. Being impressed by its useful architectural concepts many homebuilder companies as well as the individuals have been following its principles with amazing results. Thus FengShui culture has grown worldwide. The 
concerned persons in our respective fields too can follow some of its principles for the better utilization of available resources, for improving overall conditions. Before constructing a library building too we have to consider the FengShui principles that are most applicable.

\section{References:}

1. Webster, Richard - "Feng Shui for the home, Health harmony, New Delhi, 2000.
2. Pak-Tin Li and Helen Yeap - "Change your life with Feng Shui, Health harmony, New Delhi, 2000.

3. Tarkhedkar, A.R. - "Vastu Shastra (Edifice science)", vol.II, Cosmo publishing house, Nasik, 1996.

4. Speraw, Alaina, - "Basics of Feng Shui". Published : July 2002 .

5. Kemp, Kate - "Feng Phooey".

6. Internet websites. 